\title{
On stability and performance of finite control set MPC for power converters
}

by Ricardo P. Aguilera \& Daniel E. Quevedo

Copyright (C) 2011 IEEE.

This is an author-prepared version of the article, reprinted from PRECEDE 2011: Workshop on Predictive Control of Electrical Drives and Power Electronics, p. 55-62.

http://dx.doi.org/10.1109/PRECEDE.2011.6078688

This material is posted here with permission of the IEEE. Such permission of the IEEE does not in any way imply IEEE endorsement of any of University of Newcastle's products or services. Internal or personal use of this material is permitted. However, permission to reprint/republish this material for advertising or promotional purposes or for creating new collective works for resale or redistribution must be obtained from the IEEE by writing to pubs-permissions@ieee.org. By choosing to view this document, you agree to all provisions of the copyright laws protecting it. 


\title{
On Stability and Performance of Finite Control Set MPC for Power Converters
}

\author{
Ricardo P. Aguilera and Daniel E. Quevedo \\ School of Electrical Engineering and Computer Science \\ The University of Newcastle, Australia \\ Email: raguilera@ieee.org, dquevedo@ieee.org
}

\begin{abstract}
In the present work, we provide sufficient conditions for local practical stability for a specific class of power converters controlled via Finite Control Set Model Predictive Control which can be modeled as linear time-invariant systems with quantized input. In addition, we also establish bounds on the steady state behaviour of such systems. As illustrative examples, we apply our results to two converters, namely, a buck DC-DC converter, and a 2-level DC-AC inverter in a dq-coordinate frame.
\end{abstract}

\section{INTRODUCTION}

The utilization of power electronics converters has considerably increased in recent years covering a wide range of industrial applications at different rated powers (low, medium and high voltage). For this reason, power converters have been in constant development not only with respect to obtaining new advantageous architectures, but also to improve control techniques used. Here, predictive control strategies have emerged as a promising control method. Different predictive control approaches have been used to handle power converters showing that these methods hold many advantages when compared to traditional PWM control schemes [1]-[3].

In general, model predictive control (MPC) or receding horizon control [4] is a control technique which calculates the control action by solving, at each sampling instant, an optimal control problem which forecasts, over a finite horizon, the future system behaviour from the current system state. This generates an optimal control sequence. The control action to be applied to the plant is the first element of this sequence. The main advantage of MPC is that system constraints (e.g., voltage and current limitations) and non-linearities can be explicitly taken into account.

One of the most attractive predictive algorithms for power converters, is the so-called, Finite Control Set Model Predictive Control (FCS-MPC). The main advantage of this predictive strategy comes from the fact that switching actions are directly taken into account in the optimization as constraints on the system inputs. Consequently, modulation stages (to handle the switching elements) are not required. This strategy directly considers the power switches as the control input. Using a discrete model of the system, a cost function is evaluated to forecast the future behaviour of the system state at each sampling instant by exploring all the allowable switch combinations over a finite horizon. Finally, the switching action to be applied in the next sampling instant is the first element of the optimizing sequence. Despite the good performance that FCS-MPC in principle offers, there remain several open problems, being one of the most important, the stability issue. A remarkable tool to address this problem is Lyapunov stability theory [5]. This analysis can be applied to linear and non-linear systems. In the MPC case, stability can be established, by considering the cost function of the optimal problem as a candidate Lyapunov function [4], [6].

Another approach to deal with power converters is to consider them as a hybrid or switched systems [7]. In general, power converters can be described by a set of discrete states (given by switches) with an associated continuous dynamic (given by voltages and currents). Here, the different switch combinations generate a finite set of continuous-time subsystems. Thus, the control goal is achieved by commuting among these subsystems. More details related to stability of hybrid systems can be found in [8].

At times, using linear formulations of MPC may also give promising results [3], [9]. The latter approaches provide a nonquantized input (i.e. input voltage) as solution, so in practice, a modulation stage is required.

In the present work we give some insights into stability and performance of FCS-MPC. The key idea of our approach is based on representing power converters as systems with quantized inputs. This allows us to establish practical stability of the system (power converter) to a neighbourhood of a set point. As illustrative examples, the results are used to control a buck DC-DC converter and a 2-level DC-AC inverter in a dq-coordinate frame.

\section{NOTATION}

Let $\mathbb{R}$ and $\mathbb{R}_{>0}$ denote the real and non-negative real number sets. We represent the system state at the sampling instant $k$ by $x=x(k)$, and its subsequent future value as $x^{+}=$ $x(k+1)$. The transpose of a given matrix $A$ is denoted via $A^{\prime}$. The maximum and minimum eigen value of a given matrix $A$ is represented by $\lambda_{\max }(A)$ and $\lambda_{\min }(A)$ respectively. A $p$ norm is represented via $|x|_{p}$, where $p \geq 1$. $|\cdot|$ stands for the Euclidean norm. For any matrix $\mathrm{P},|x|_{P}^{2}=x^{\prime} P x$ represents the square weighted Euclidean norm. We define a neighbourhood of the origin as a ball via $\mathcal{B}_{\delta}=\left\{x \in \mathbb{R}^{n}:|x|<\delta\right\}$, where $\delta$ is a positive number. The operator $q\{\cdot\}$ and the variable $\eta$ stand for the quantization function and quantization noise respectively, which satisfy $q\{x\}=x+\eta$ and $|\eta| \leq \Delta_{q}$, where $\Delta_{q}>0$ stands for maximum quantization error. 


\section{Preliminaries}

A key observation on power converters is that since system inputs (switch combinations) are restricted to belong to a finite set, in general, the best one can hope for, is that state trajectories be bounded around the desired system reference. Therefore, in the present work we will focus on practical stability or ultimate boundedness. The practical term is used to emphasize that only stability of a neighbourhood of the system reference can be guaranteed. To understand this concept, some useful definitions are provided below (for further background, see Section 5.2 in [5] ).

\section{A. Basic Definitions}

Consider the discrete-time system described by:

$$
x^{+}=f(x, u),
$$

where $x \in \mathbb{X} \subset \mathbb{R}^{n}$ is the system state and $u \in \mathbb{U} \subset \mathbb{R}^{m}$ is the control input. We assume that the origin is an equilibrium point for (1). Thus, $f(0,0)=0$.

Definition 1: (Control positive invariant set). A set $\mathcal{A} \subseteq \mathbb{R}^{n}$ is said to be a control positive invariant set (PIS) for the system (1) if there exists an input $u \in \mathbb{U}$ such that $f(x, u) \in \mathcal{A}$, for all $x \in \mathcal{A}$.

Definition 2: (Practical Asymptotical Stability) The system (1) is said to be practically Asymptotically Stable (AS) in $\mathcal{A} \subseteq$ $\mathbb{R}^{n}$ if $\mathcal{A}$ is a PIS for (1) and if there exists a positive constant $\delta$, and a monotonically decreasing function in $k, \beta\left(x_{0}, k\right)$ such that

$$
|x(k)| \leq \beta\left(x_{0}, k\right)+\delta,
$$

for all $x \in \mathcal{A}$ and $k \geq 0$. Here, $|x(k)|$ could be any norm of the system state.

In the case that the function $\beta\left(x_{0}, k\right)$ is given by $\beta\left(x_{0}, k\right)=$ $c\left|x_{0}\right|^{l} \rho^{k}$, where $c \geq 1, \rho \in(0,1)$ and $l>0$, we will say that system (1) is practically Exponentially Stable (ES) in $\mathcal{A}$. Thus, (2) can be expressed by:

$$
|x(k)|^{l} \leq c\left|x_{0}\right|^{l} \cdot \rho^{k}+\delta,
$$

for all $x \in \mathcal{A}$ and $k \geq 0$.

Definition 3: (Ultimate invariant set) Notice from (2) that if system (1) is AS, since the function $\beta\left(x_{0}, k\right)$ decreases over time, $\beta\left(x_{0}, k\right) \rightarrow 0$ as $k \rightarrow \infty$, the system state will ultimately be confined in

$$
\mathcal{D}_{\delta}=\left\{x \in \mathbb{R}^{n}:|x(k)| \leq \delta\right\} .
$$

In other words, one can say that the set $\mathcal{D}_{\delta}$ is stable for the system (1). This situation is depicted in Fig. 1.

\section{B. Practical Lyapunov Stability}

To understand Lyapunov stability theory one can establish an analogy of this kind of stability to the energy behaviour in a passive electrical system. For example, in an RLC circuit, the total stored energy is the sum of individual stored energy in the inductor, $E_{L}=\frac{1}{2} L i_{L}^{2}$, and capacitor, $E_{C}=\frac{1}{2} C v_{c}^{2}$. This energy is dissipated in the resistance $\mathrm{R}$ as the time proceeds.
Thus, the total system energy, $E_{T}=E_{L}+E_{C}$, will decay to zero. Consequently, the system state $\left(i_{L}, v_{c}\right)$ tends to a final equilibrium point, $i_{L}=v_{c}=0$. The Lyapunov theory follows a similar concept. If one can find a function for a given system which evolves over time similarly to the energy in a passive system, then the system state will converge to an equilibrium point. From this intuitive idea, we next give a simplified discussion of this theory. See [5] for a more thorough presentation.

Definition 4: (Practical control-Lyapunov Function) A continuous function $V: \mathbb{R}^{n} \rightarrow \mathbb{R}_{\geq 0}$ is said to be a practical control-Lyapunov function (CLF) in $\mathcal{A} \subseteq \mathbb{R}^{n}$ for the system (1) if $\mathcal{A}$ is a control PIS and if there exists a compact set (i.e., closed and bounded) $\Omega \subseteq \mathcal{A}$, some positive constants $a_{1}, a_{2}$, $a_{3}$ and $\gamma$ such that

$$
\begin{aligned}
V(x) & \geq a_{1}|x|^{l}, & & \forall x \in \mathcal{A}, \\
V(x) & \leq a_{2}|x|^{l}, & & \forall x \in \Omega, \\
V(f(x, u))-V(x) & \leq-a_{3}|x|^{l}+\gamma, & &
\end{aligned}
$$

for all $x \in \mathcal{A}, u \in \mathbb{U}$ and $l \geq 1$.

Theorem 1: (Converse theorem for asymptotic stability) If the system (1) admits a practical control-Lyapunov function in $\mathcal{A}$, then it is asymptotically stable in $\mathcal{A}$.

The above result tells us that if we can find a CLF for the system to be controlled, then it is a sufficient condition to guarantee asymptotic (exponential) stability of that system as presented in Definition 2. In other words, a practical CLF provides sufficient conditions for the existence of a controller $u=\kappa(x)$ which ensures asymptotic (exponential) stability to the neighbourhood $\mathcal{D}_{\delta}$ for the controlled system $x^{+}=f(x, \kappa(x))$.

\section{Practical Stability of MPC}

There exist several MPC methodologies in the literature, which have some common ingredients that allow one to formalize this field (see [10]). The main common ingredient is the fact that these predictive strategies rely on a cost function to forecast the future behaviour of the system to be controlled.
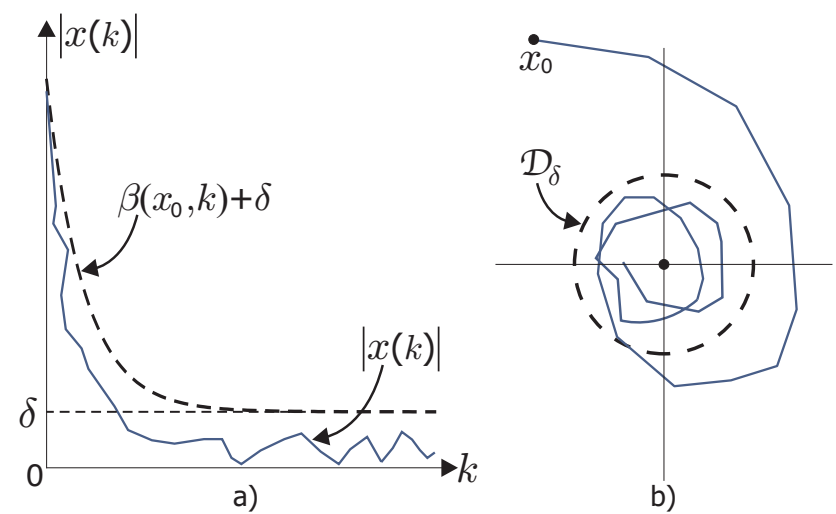

Fig. 1. Practical stability representation: a) Practical asymptotical (exponential) stability. b) Ultimate invariant set $\mathcal{D}_{\delta}$. 
Hence, a key aspect to establish stability of MPC is based on considering this cost function as a candidate-Lyapunov function. Thus, stability analysis generally focuses on showing that such cost function satisfies the conditions presented in Definition 4.

In general, a cost function, with a prediction horizon $N$, can be expressed by:

$$
V_{N}(x(k), \vec{u})=V_{f}(x(N))+\sum_{k=0}^{N-1} \ell(x(k), u(k)) .
$$

The term $\ell(x(k), u(k))$ in (7) is called the stage cost. This term is used to define a desired predicted evolution of the system state and the input. On the other hand, we have the term called terminal cost, which is represented by $V_{f}(x(N))$. This term is designed, based on the stage cost, in order to guarantee the system convergence to the reference. To do this, we first recall that the system state is normally constrained (due to voltage and current limitations) to $x \in \mathbb{X}$. In addition, we will impose an extra constraint for the last predicted state, called terminal constraint

$$
x(N) \in \mathbb{X}_{f} \subseteq \mathbb{X}
$$

We define $X_{N}$ as the domain of the cost function. Thus, the set $X_{N}$ is a subset of $\mathbb{X}$ which includes all the system states, $x \in \mathbb{X}$, that can be led to the terminal region, $\mathbb{X}_{f}$, in $N$ steps in order to satisfy (8).

To establish stability of the MPC strategy, we introduce the following condition:

Assumption 1: (Practical Stability Condition) The cost function, $V(x)$, is designed such that there exist a $V_{f}(x)$ and a local controller $u_{f}(x)$ which satisfy the following condition in the terminal region:

$$
V_{f}\left(f\left(x, u_{f}(x)\right)\right)-V_{f}(x)+\ell\left(x, u_{f}(x)\right)<\gamma, \quad \forall x \in \mathbb{X}_{f},
$$

for a given $\gamma \geq 0$.

Theorem 2: If MPC strategy satisfies that $x(N) \in \mathbb{X}_{f}$ and if Assumption 1 holds, then the system (1) under MPC is practical asymptotically stable.

The proof of this theorem can be found in [6]. A more rigorous analysis of stability of MPC with finite control sets is given in [11].

This theorem implies that (9) is a sufficient condition to guarantee that the cost function is a practical CLF, as presented in Definition 4. This amounts to designing $V_{f}(x)$ and a suitable local control policy $u_{f}(x)$.

Finally, if the cost function, $V(x)$, presented in (7) is designed satisfying condition (9), we can say that the system (1) is practically asymptotically stable.

\section{Finite-CONTROL-SET MPC}

In this section we will investigate FCS-MPC. We will use Lyapunov stability theory to design a suitable cost function in order to guarantee practical stability of this strategy when power converters can be modeled as linear time-invariant (LTI) systems with a quantized input.

\section{A. Problem Definition}

Consider that the power converter to be controlled can be modeled, in a state space framework, via:

$$
x^{+}=f(x, u)=A x+B u,
$$

where $x \in \mathbb{X} \subseteq \mathbb{R}^{n}$ stands for the $n$-system state variables (i.e. voltages and currents) and $u \in \mathbb{U} \subset R^{m}$ represents the $m$-control inputs (i.e. switches or input voltage levels) of the power converter. In this case, due to the nature of the system, we deal with a linear system wich presents a finite control set of $q$ elements, such that

$$
u \in \mathbb{U}=\left\{u_{1}, \ldots, u_{q}\right\} .
$$

In particular, we are interested in applying FCS-MPC strategy to the system (10). In this case we will focus in a quadratic cost function, with a prediction horizon $N$, defined via:

$$
V_{N}(x, \vec{u})=|x(N)|_{P}^{2}+\sum_{k=0}^{N-1}|x(k)|_{Q}^{2}+|u(k)|_{R}^{2} .
$$

comparing to the form given in (7) we have that,

$$
\ell(x, u)=|x|_{Q}^{2}+|u|_{R}^{2}=x^{\prime} Q x+u^{\prime} R u,
$$

is the stage cost, in which $Q$ and $R$ are positive definite matrices, and

$$
V_{f}(x)=|x|_{P}^{2}=x^{\prime} P x,
$$

in the final cost, in which $P$ is also positive definite.

Remark 1: It is important to notice that $Q$ and $R$ are weighting matrices used to penalize predicted behaviour. Hence, these are given matrices. On the other hand, matrix $P$ is used to penalize the final predicted state in order to satisfy (9). The design of matrix $P$ will be analyzed in Section IV.

Now, the FCS-MPC strategy can be stated as an optimal control problem, $\mathbb{P}_{N}(x)$, for the system (10), as:

$$
\mathbb{P}_{N}(x): \quad V_{N}^{0}(x)=\min _{\vec{u}}\left\{V_{N}(x, \vec{u}) \mid \vec{u} \in \mathcal{U}_{N}(x)\right\},
$$

where $\mathcal{U}_{N}(x)$ is the set of control sequences, $\vec{u}$, which satisfies

$$
\begin{aligned}
x(k) & \in \mathbb{X}, \quad \forall k \in\{0, \cdots, N\}, \\
u(k) & \in \mathbb{U}, \quad \forall k \in\{0, \cdots, N-1\}, \\
x(N) & \in \mathbb{X}_{f} .
\end{aligned}
$$

The domain of the cost function is expressed by:

$$
X_{N}=\left\{x \in \mathbb{X}: \mathcal{U}_{N}(x) \neq \emptyset\right\} .
$$

To apply this strategy, at the initial sampling instant, $k=0$, we take a measurement of the system state, $x=x(0)$. Since the system input, $u$, belongs to the finite set $\mathbb{U}$, we can evaluate the cost function for all possible inputs over the prediction horizon $N$. The result of this optimization will give us an optimal input sequence, $\vec{u}$, defined by:

$$
\vec{u}_{\text {op }}=\{u(0), \cdots, u(N-1)\} .
$$

Remark 2: It is important to emphasize that $\vec{u}_{o p}$ satisfies all the system constraints given by (14)-(16) which are normally 
related to a safe operation of the power converter (i.e. voltage and currents limits) and the finite available choices (i.e. switches or voltage levels).

Finally, the optimal input, $u_{o p}$, to be applied to the system is only the first element of $\vec{u}_{o p}$

$$
u_{o p}=u(0) \triangleq \kappa_{N}(x),
$$

discarding the remaining elements. Consequently, the system state in the next sampling instant, $k=1$, will be

$$
x^{+}=x(1)=A x+B \kappa_{N}(x) .
$$

Afterwards, this process is repeated starting from this new initial point.

\section{B. FCS-MPC of a Buck Converter}

To illustrate the above, we will apply FCS-MPC to a buck DC-DC converter (see Fig. 2). This topology has 3 switches. Each of them can present only two values, i.e., $S_{i}=0$ if the switch is open and $S_{i}=1$ when it is closed. It means that $S_{i} \in\{0,1\}$, for all $i \in\{1,2,3\}$. It is clear that, for avoiding internal faults, some of the switch combinations are forbidden. Thus, if we define the switching input vector as: $s(t)=\left[\begin{array}{lll}S_{1}(t) & S_{2}(t) & S_{3}(t)\end{array}\right]^{\prime}$, then it will be restricted to belong to the following set:

$$
s(t) \in\left\{\left[\begin{array}{l}
0 \\
0 \\
1
\end{array}\right], \quad\left[\begin{array}{l}
0 \\
1 \\
0
\end{array}\right], \quad\left[\begin{array}{l}
1 \\
0 \\
0
\end{array}\right]\right\} .
$$

This is equivalent to consider the input voltage, $v_{i}(t)$, as control input, which is constrained according to:

$$
v_{i}(t) \in\left\{0, \quad \frac{V_{d c}}{2}, \quad V_{d c}\right\} .
$$

Thus, the continuous-time model for the buck DC-DC converter is expressed via:

$$
\begin{aligned}
& \frac{d i_{L}(t)}{d t}=-\frac{1}{L} v_{0}(t)+\frac{1}{L} v_{i}(t), \\
& \frac{d v_{0}(t)}{d t}=\frac{1}{C} i_{L}(t)-\frac{1}{r C} v_{0}(t) .
\end{aligned}
$$

Now, we assume that we seek to obtain an output voltage of $v_{0}^{\star}=\alpha V_{d c}$, with $\alpha \in(0,1)$. From the system model, one can see that this voltage reference will be reached when the inductor current is $i_{L}^{\star}=v_{0}^{\star} / r=\alpha V_{d c} / r$. On the other hand, the required voltage input, $v_{i}^{\star}$, to keep this desired steady state is $v_{i}^{\star}=v_{0}^{\star}=\alpha V_{d c}$.

Remark 3: Notice that the desired output reference, $v_{0}^{\star}$, may not be an element of the available inputs in (18). Therefore, it is not possible to achieve an equilibrium point for some references. Thus, the best one can hope for, is that state trajectories be bounded around the desired reference. It is for this reason we focus on practical stability as shown in Fig. 1.

To facilitate the analysis, we next study the power converter in per-unit frame. To do this, a base voltage, $V_{b a s e}=V_{d c}$, and a base current, $I_{b a s e}=V_{d c} / r$, are considered. Afterwards, using a forward Euler approximation, we transform the

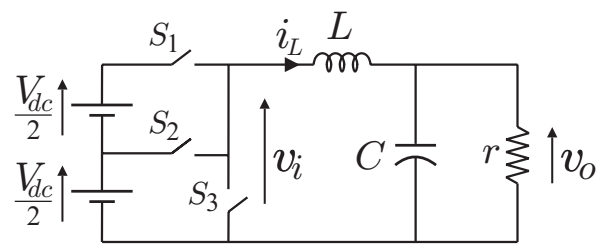

Fig. 2. 3-level buck DC-DC converter.

continuous-time model in (19) into discrete-time form. Thus, the buck DC-DC converter in per unit frame, can be expressed by:

$$
\begin{aligned}
i_{L}(k+1) & =i_{L}(k)-\frac{h r}{L} v_{0}(k)+\frac{h r}{L} v_{i}(k), \\
\left.v_{0}^{(} k+1\right) & =\frac{h}{r C} i_{L}(k)+\left(1-\frac{h}{r C}\right) v_{0}(k),
\end{aligned}
$$

where $h$ stands for the sampling period.

Now, we define the state and input deviations as:

$$
x=\left[\begin{array}{l}
i_{L}-\alpha \\
v_{0}-\alpha
\end{array}\right], \quad u=v_{i}-\alpha .
$$

Finally, the system deviation between the system state and the desired reference, in per-unit frame, is characterized via

$$
x^{+}=A x+B u,
$$

where

$$
A=\left[\begin{array}{cc}
1 & -\frac{h r}{L} \\
\frac{h}{r C} & 1-\frac{h}{r C}
\end{array}\right], \quad B=\left[\begin{array}{c}
\frac{h r}{L} \\
0
\end{array}\right] .
$$

Consider that, for safety reasons, it is required to operate the converter under the following conditions: $0<i_{L}<i_{\max }$ and $0<v_{0}<v_{\max }$. Hence, the state deviation, $x$, is restricted to belong to the set $\mathbb{X}$ defined by:

$$
\mathbb{X}=\left\{x \in \mathbb{R}^{2}: x \in\left[\begin{array}{ll}
-\alpha, & i_{\max }-\alpha \\
-\alpha, & v_{\max }-\alpha
\end{array}\right]\right\},
$$

while the control input, $u$, is restricted to belong to the finite set $\mathbb{U}$ defined via:

$$
\mathbb{U}=\left\{u \in \mathbb{R}: u \in\left\{-\alpha, \quad \frac{1}{2}-\alpha, \quad 1-\alpha\right\}\right\} .
$$

To design the cost function, we define the weighting factor $\omega_{x}$ which gives us a trade off between the inductor current tracking error, $x_{1}$, and the output voltage tracking error, $x_{2}$. In addition, a weighting factor for the control input $\omega_{u}$ is also defined. Thus, the quadratic cost function (12) for the buck DC-DC converter is chosen with parameters:

$$
Q=\left[\begin{array}{cc}
1 & 0 \\
0 & \omega_{x}
\end{array}\right], \quad R=\omega_{u}
$$

The remaining design of the terminal cost (matrix $P$ ) is analyzed in the following section. 


\section{LYAPUNOV-BASED PERFORMANCE ANALYSIS OF FCS-MPC}

To guarantee practical stability when FCS-MPC is applied to system (10), by Theorem 1, it is sufficient to prove that $V(x)$ satisfies the conditions (4)-(6) presented in Definition 4. Thus, the cost function can be considered as a practical CLF. As shown in Section II-C, this task can be carried out by finding $u_{f}(x)$ and $V_{f}(x)$ which satisfy Assumption 1. Consequently, the system (10) controlled by FCS-MPC will be practically asymptotically (exponentially) stable.

Given the nature of our problem, and in view for results for unconstrained systems, see [6], we choose $u_{f}(x)$ and $V_{f}(x)$ as follows:

$$
u_{f}(x)=q\{K x\} .
$$

Thus, system (10), in the terminal region, $\mathbb{X}_{f}$, can be modeled by:

$$
x^{+}=A_{K} x+B \eta, \quad \forall x \in \mathbb{X}_{f},
$$

where $A_{K}=A+B K$ and $\eta$ stands for the quantization error. Then, we analyze the practical stability condition (9) for our problem.

$$
\begin{aligned}
V_{f} & \left(A x+B u_{f}\right)-V_{f}(x)+\ell\left(x, u_{f}\right) \\
\quad & =\left|A_{K} x+B \eta\right|_{P}^{2}-|x|_{P}^{2}+|x|_{Q}^{2}+|K x+\eta|_{R}^{2}, \\
& =x^{\prime}\left(A_{K}^{\prime} P A_{K}+Q^{*}-P\right) x+\eta^{\prime}\left(B^{\prime} P B+R\right) \eta \\
& +2 x^{\prime}\left(A_{K}^{\prime} P B+K^{\prime} R\right) \eta,
\end{aligned}
$$

where $Q^{*}=Q+K^{\prime} R K$. Here, the matrix $P$ is chosen to be the solution to the discrete Riccati equation

$$
A_{K}^{\prime} P A_{K}-P+Q^{*}=0,
$$

in which

$$
K=-\left(B^{\prime} P B+R\right)^{-1} B^{\prime} P A .
$$

In addition, the following relationship can be derived

$$
A_{K}^{\prime} P B+K^{\prime} R=A^{\prime} P B+K^{\prime}\left(B^{\prime} P B+R\right)=0 .
$$

Consequently, considering that the quantization error is bounded by $|\eta| \leq \Delta_{q}$, we have that

$V_{f}\left(A x+B u_{f}\right)-V_{f}(x)+\ell\left(x, u_{f}\right) \leq\left|B^{\prime} P B+R\right| \Delta_{q}^{2}, \forall x \in \mathbb{X}_{f}$

Now, we can say that, the proposed quadratic cost function, $V(x)$, presented in (12), is a practical CLF, satisfying that:

$$
\begin{aligned}
V(x) & >a_{1}|x|^{2}, \quad \forall x \in X_{N} \\
V(x) & <a_{2}|x|^{2}, \quad \forall x \in \mathbb{X}_{f} \\
\Delta V(x) & <-a_{3}|x|^{2}+\gamma,
\end{aligned}
$$

for all $x \in X_{N}$, where

$$
\begin{aligned}
a_{1}=a_{3} & =\lambda_{\min }(Q), \\
a_{2} & =\lambda_{\max }(P), \\
\gamma & =\left|B^{\prime} P B+R\right| \Delta_{q}^{2} .
\end{aligned}
$$

Hence, system (10) is practically exponentially stable.

Notice that the cost function, $V(x)$, will monotonically decrease, until the system state reaches a value of $|x|^{2}<\frac{\gamma}{a_{3}}$.
It follows from (29), we have that

$$
V\left(x^{+}\right) \leq V(x)-a_{3}|x|^{2}+\gamma,
$$

considering that $V(x)<a_{2}|x|^{2}$, we can derive the following bound for the cost function:

$$
V(x(k)) \leq V\left(x_{0}\right) \cdot \rho^{k}+\gamma,
$$

where

$$
\rho=1-\frac{a_{3}}{a_{2}}<1
$$

is the decay rate.

Considering that $V(x)>a_{1}|x|^{2}$ we have that

$$
|x(k)| \leq \frac{1}{a_{1}} V\left(x_{0}\right) \cdot \rho^{k}+\frac{\gamma}{a_{1}},
$$

Thus, as presented in Definition 3, one obtain the following ultimate invariant set for the quadratic cost function via:

$$
\mathcal{D}_{\delta}=\left\{x \in \mathbb{R}^{n}:|x(k)|^{2} \leq \delta\right\} .
$$

where

$$
\delta=\frac{\gamma}{a_{1}}=\frac{\left|B^{\prime} P B+R\right|}{\lambda_{\min }(Q)} \Delta_{q}^{2} .
$$

Remark 4: It is important to emphasize that the suboptimal controller $u_{f}=q\{K x\}$ is not implemented. It is only used to establish stability and to define the ultimate invariant set $\mathcal{D}_{\delta}$. Thus, it is not necessary to chose $K$ as presented in (28). One can use any matrix $K$ which produces a stable matrix $A_{K}$ $\left(\lambda\left(A_{K}\right)<1\right)$. Then, one can obtain a positive definite matrix $P$ by solving the Lyapunov equation (27).

\section{Simulation AnAlysis}

In this section we illustrate the Lyapunov-based stability analysis presented in this work when it is applied to two class of power converters, a buck converter and a 2-level inverter.

\section{A. Buck DC-DC converter}

To verify the performance of the FCS-MPC strategy applied to the buck converter, simulation studies were carried out by using the mathematical model presented in Section III-B. The electrical parameters of this DC-DC converter, presented in Fig. 2, are chosen as $V_{d c}=100[V], r=10[\Omega], L=10[m H]$ and $C=200[\mu F]$. The desired output voltage reference is set as $v_{o}^{\star}=37.5[V]$. It means that $\alpha=0.375$.

The predictive controller was implemented using a sampling period of $h=200[\mu s]$. In addition, the cost function is set considering $\omega_{x}=1$ and $\omega_{u}=0.1$. Thus, the cost stage is set, as was presented in (24), via:

$$
Q=\left[\begin{array}{ll}
1 & 0 \\
0 & 1
\end{array}\right], \quad R=0.1
$$

Afterwards, we can solve the Riccati equation presented in (27) along with the controller gain equation (28), in order to set the terminal cost. Thus, we obtain that:

$$
P=\left[\begin{array}{cc}
3.2271 & -0.2591 \\
-0.2591 & 1.0563
\end{array}\right], \quad K=\left[\begin{array}{ll}
-2.5912 & 0.5635
\end{array}\right] \text {. }
$$


Finally, considering a prediction horizon of $N=1$, the cost function (12) becomes:

$$
V_{N}(x, \vec{u})=|x(k)|_{Q}^{2}+|u(k)|_{R}^{2}+|x(k+1)|_{P}^{2} .
$$

From this design, we can say that the cost function will be exponentially bounded as shown in (34), with a decay rate of:

$$
\rho=1-a_{2} / a_{3}=0.6930,
$$

until reaching a final value of

$$
\gamma=\left|B^{\prime} P B+R\right| \Delta_{q}^{2}=0.0573 .
$$

Thus, one can anticipate that the system state deviation, $x$, will be led by the predictive controller to the ultimate invariant set, $\mathcal{D}_{\delta}$, characterized via:

$$
\mathcal{D}_{\delta}=\left\{x \in \mathbb{R}^{n}:|x| \leq \delta=0.2394\right\} .
$$

The evolution of the buck converter under FCS-MPC, starting from $v_{0}=i_{L}=0$, is depicted in Fig. 3. Here, one can see that the predictive controller leads the system state to the ultimate invariant set $\mathcal{D}_{\delta}$. The system state (inductor current $i_{L}$ and output voltage $v_{o}$ ) and the finite control input (input voltage $v_{i}$ ) behaviour, over the time, are shown in Fig. 4. It is clear that the system trajectories are bounded around of the desired reference.

Due to the design of the cost function, $V(x)$, one can see in Fig. 5 that, during the whole trajectory, $V(x)$ is exponentially bounded as defined in (34). Hence, it decreases exponentially until the system state deviation, $x$, reaches the ultimate invariant set $\mathcal{D}_{\delta}$. Inside this region, the cost function, $V(t)$, presents an oscillating behaviour bounded by $\gamma$. This is attributable to the fact that the system cannot reach an equilibrium point for the given reference.

To show how the cost function design affects the system behaviour, we carried out simulations for the buck converter using a different matrix $R$. In this case we proposed to set matrix $R=0.01$. Following the same procedure used previously, we obtain

$$
P=\left[\begin{array}{ll}
2.22401 & -0.0441 \\
-0.0441 & 1.00090
\end{array}\right], \quad K=\left[\begin{array}{ll}
-4.4057 & 0.8990
\end{array}\right] \text {. }
$$

Consequently, under this new setting the cost function satisfies the bound (34) with a decay factor $\rho=0.5507$ and a final value of $\gamma=0.0247$.

Finally, the ultimate invariant set for the system state deviation under this new scenario is given by:

$$
\mathcal{D}_{\delta}=\left\{x \in \mathbb{R}^{n}:|x| \leq \delta=0.1572\right\} .
$$

The system state deviation behaviour, $x$, under this new cost function setting, is presented in the Figures 6 and 7. Here one can observe that the system trajectories produce a smaller tracking error than in the previous case. This is achieved by incrementing the commutations between the available inputs. On the other hand, in Fig. 8 one can clearly see that the cost function decrease faster than in the previous case.

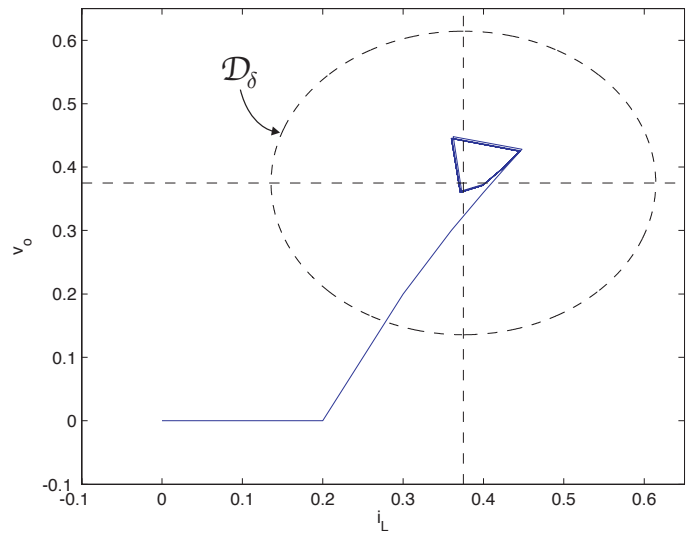

Fig. 3. Convergence of the buck converter to the ultimate invariant set $\mathcal{D}_{\delta}$. $\delta=0.2394$.
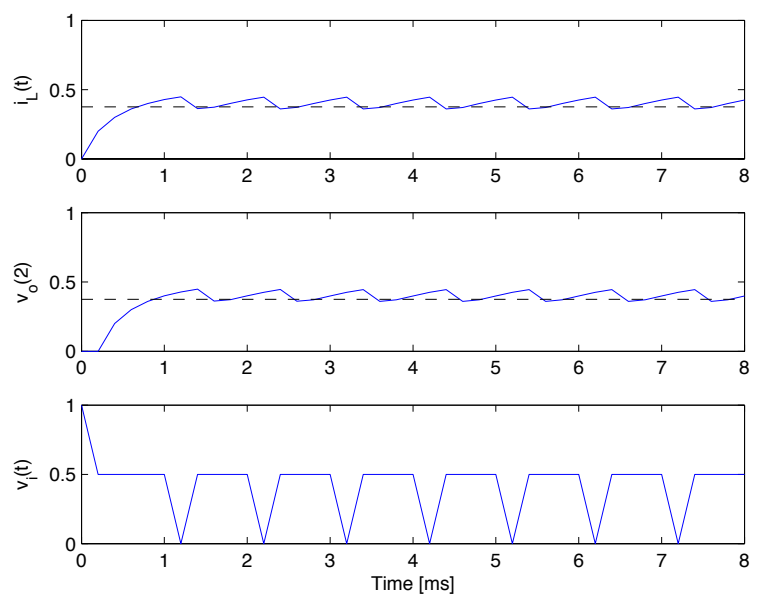

Fig. 4. System state and input behaviour over time.
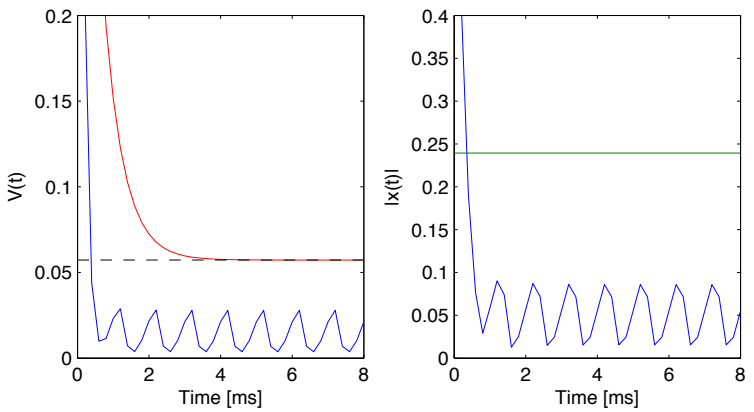

Fig. 5. Cost function $V(t)$ and its exponentially bound; Norm of the state deviation $|x|$ over time.

\section{B. 2-Level Inverter}

Here, we study the performance of FCS-MPC when it is applied to the 2-level AC-DC converter presented in Fig. 9.

The continuous-time dynamic model for each output current, $i_{y}$, is given by:

$$
\frac{d i_{y}}{d t}=-\frac{r}{L}+\frac{1}{L}\left(v_{i y}-v_{n o}\right)
$$




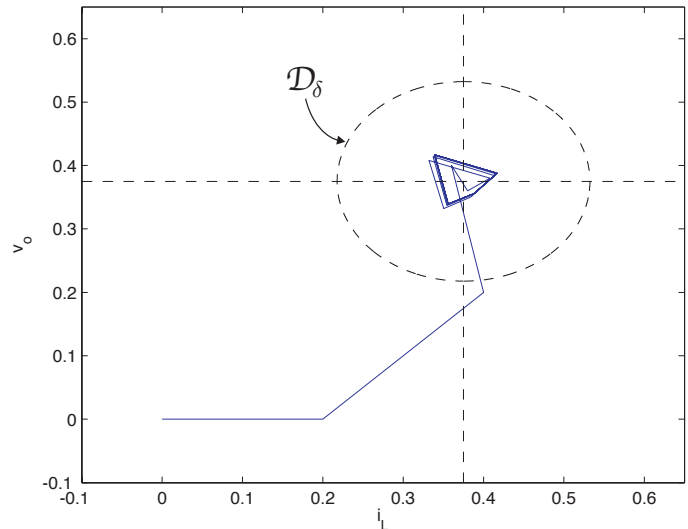

Fig. 6. Convergence of the buck converter to the ultimate invariant set $\mathcal{D}_{\delta}$. $\delta=0.1572$.
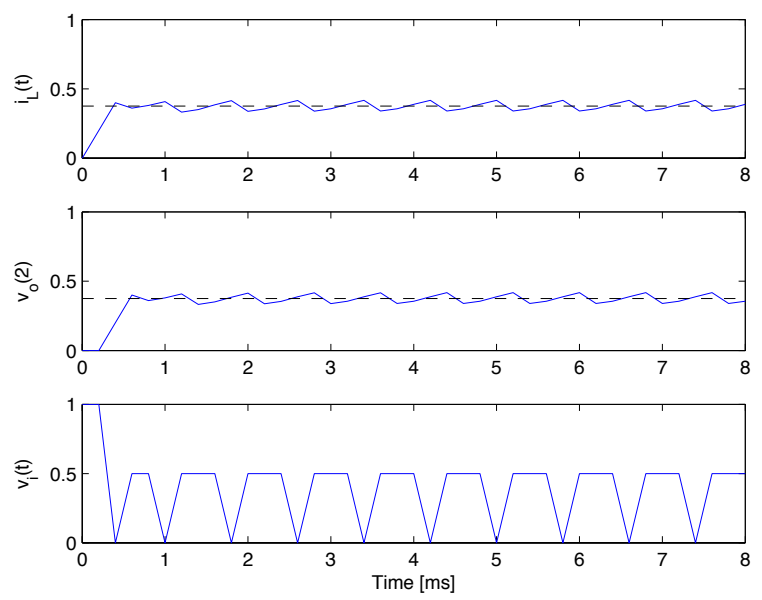

Fig. 7. System state and input behaviour over time.
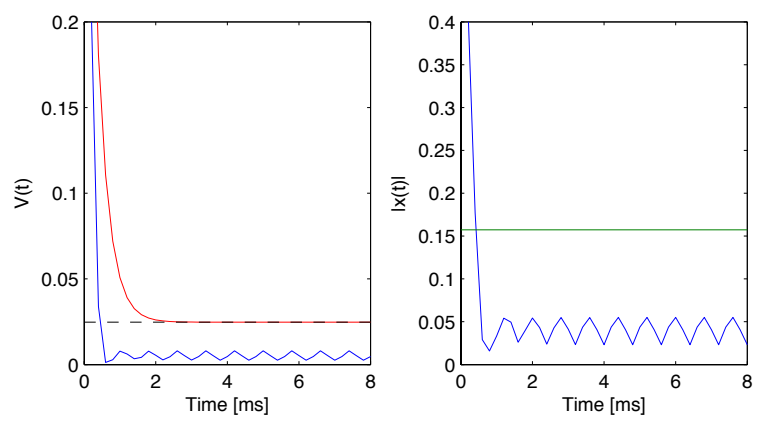

Fig. 8. Cost function $V(t)$ and its exponentially bound $\hat{V}_{b}$; Norm of the state deviation $|x|$ over time.

for all $y \in\{a, b, c\}$. Here, $v_{n o}$ stands for the common mode voltage defined as $v_{n o}=\frac{1}{3}\left(v_{i a}+v_{i b}+v_{i c}\right)$.

In this case, the input, $v_{i y}$, is restricted to take only two values

$$
v_{i y} \in\left\{0, V_{d c}\right\}, \quad \forall y \in\{a, b, c\},
$$

It is well known that, for sinusoidal references in a 3-phase system, we can apply the so-called abc to dq transform. Firstly,

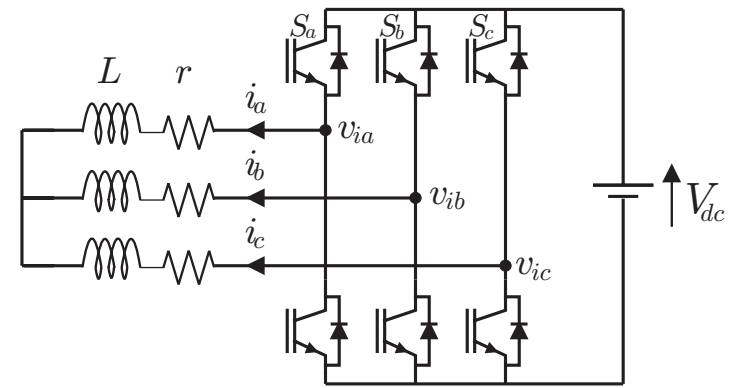

Fig. 9. 2-Level inverter topology.

we define a current vector in abc frame as: $i_{a b c}=\left[\begin{array}{lll}i_{a} & i_{b} & i_{c}\end{array}\right]^{\prime}$. Then, it is transform into dq frame by applying the following transformation:

$$
i_{d q}=\Gamma(t) i_{a b c}
$$

where:

$$
\Gamma(t)=\left[\begin{array}{lll}
\sin (\omega t) & \sin (\omega t-2 \pi / 3) & \sin (\omega t+2 \pi / 3) \\
\cos (\omega t) & \cos (\omega t-2 \pi / 3) & \cos (\omega t+2 \pi / 3)
\end{array}\right]
$$

and $i_{d q}=\left[\begin{array}{ll}i_{d} & i_{q}\end{array}\right]^{\prime}$.

Thus, the continuous-time model of the 2-level inverter, in dq frame, is expressed by:

$$
\frac{d i_{d q}}{d t}=\left[\begin{array}{cc}
-r / L & \omega \\
-\omega & -r / L
\end{array}\right] i_{d q}+\frac{1}{L} \Gamma(t) v_{a b c}
$$

where $v_{a b c}=\left[\begin{array}{lll}v_{i a} & v_{i b} & v_{i c}\end{array}\right]^{\prime}$ is the input vector.

Similar to the buck converter, we define the state and input deviations, $x$, for the 2-level inverter. In this case we seek a constant amplitud reference, $I^{\star}$, for the output currents $i_{a b c}$. This is equivalent to set $i_{d}^{\star}=I^{\star}$ and $I_{q}^{\star}=0$. Thus, the system state deviation in dq frame is characterized via:

$$
x=\left[\begin{array}{c}
i_{d}-I^{\star} \\
i_{q}
\end{array}\right], \quad u=\Gamma(t) v_{a b c}-\left[\begin{array}{c}
r I^{\star} \\
\omega L I^{\star}
\end{array}\right],
$$

Then, using forward Euler approximation, we obtain a discrete-time model, for the system deviation, expressed by:

$$
x^{+}=A x+B u,
$$

where

$$
A=\left[\begin{array}{cc}
1-h \frac{r}{L} & \omega L \\
-\omega L & 1-h \frac{r}{L}
\end{array}\right], \quad B=\left[\begin{array}{cc}
\frac{h}{L} & 0 \\
0 & \frac{h}{L}
\end{array}\right] .
$$

The electrical parameters used for the simulation analysis are $V_{d c}=400[V], r=10[\Omega]$ and $L=30[\mathrm{mH}]$. The desired amplitude for the output current is $I^{\star}=5[A]$ with a frequency of $f_{0}=50[\mathrm{~Hz}]$. The cost function was set as

$$
Q=\left[\begin{array}{ll}
1 & 0 \\
0 & 1
\end{array}\right], \quad R=0_{2 \times 2} .
$$

In this case we also set the controller gain as:

$$
K=\left[\begin{array}{cc}
-0.1015 & -0.0033 \\
0.0033 & -0.1015
\end{array}\right] \text {. }
$$




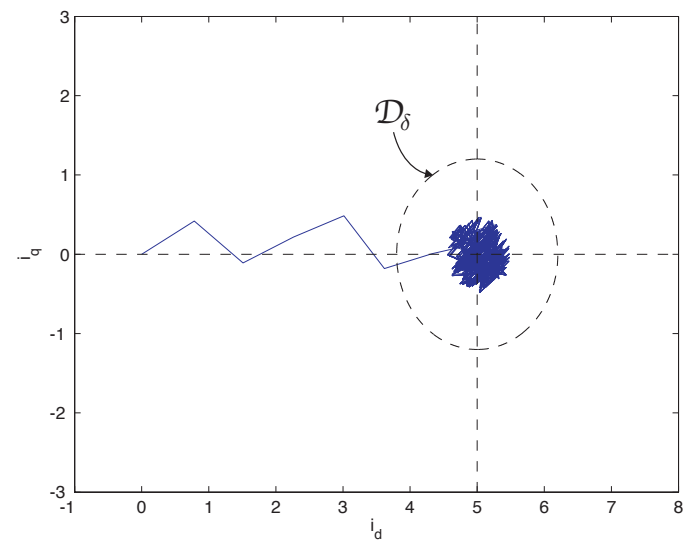

Fig. 10. Convergence of the 2-level inverter in dq frame to the ultimate invariant set $\mathcal{D}_{\delta} . \delta=1.2009$.
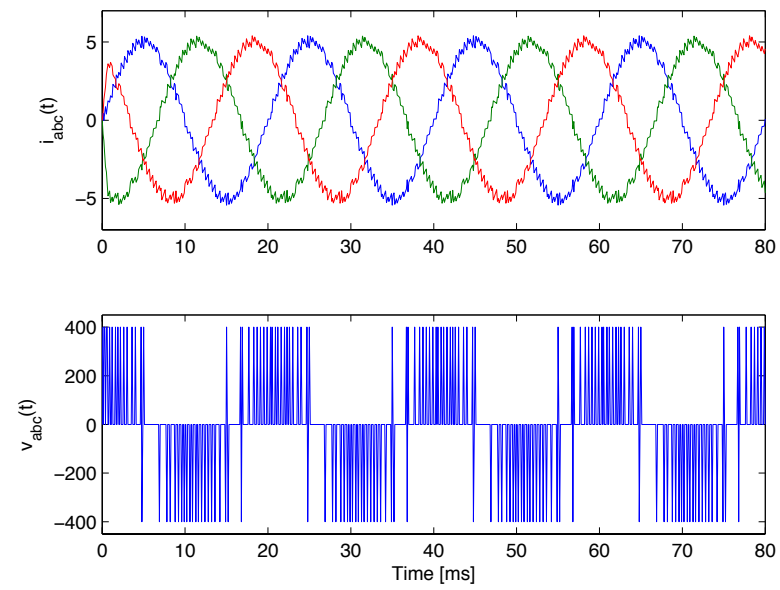

Fig. 11. Output current and inverter voltage in abc frame.
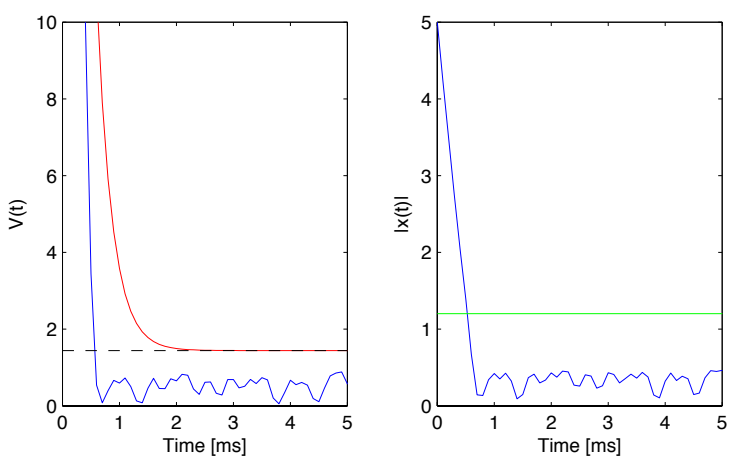

Fig. 12. Cost function $V(t)$ and its exponentially bound; Norm of the state deviation $|x(t)|$ over the time.

Thus, we obtain, by solving the Lyapunov equation (27), a diagonal $P$ matrix defined by:

$$
P=\left[\begin{array}{cc}
3.2451 & 0 \\
0 & 3.2451
\end{array}\right]
$$

Consequently, the cost function satisfies the bound (34) with a decay factor $\rho=0.6918$ and a final value of $\gamma=1.4423$. Thus, the ultimate invariant set, where the system state deviation, $x$, will be confined, is given by:

$$
\mathcal{D}_{\delta}=\left\{x \in \mathbb{R}^{n}:|x| \leq \delta=1.2009\right\} .
$$

The behaviour of the state deviation of the 2-level inverter is shown in Fig. 10. Here, the controller leads the system to the desired reference, in terms of amplitud. (dq frame). Consequently, this yields a sinusoidal behaviour of the output currents, $i_{a b c}$, as it is depicted in Fig. 11. On the other hand, the evolution of the cost function, $V(x(t))$, is shown in Fig. 12. This is exponentially bounded by (34). A similar behaviour can be observed in the norm of the system state $|x(t)|$.

\section{CONCLUSIONS}

When controlling solid-state power converters in discretetime, in general, voltages and currents will not converge to the desired steady-state values. This motivates the analysis of such converters from a practical stability viewpoint, i.e., by analysing convergence of state variables to a bounded invariant set. The present work has used Lyapunov-stability concepts to study local practical stability of Finite-Set MPC when applied to a class of power converters. Our results show how the cost function can be designed to guarantee practical stability. We also provide bounds on the associated invariant sets. Future work may include elucidating the trade-off that exists when designing the local control policy $u_{f}(x)$ and the horizon length $N$, and the size of the invariant set and the region of attraction of the loop. It is also of interest to study other converter topologies and to confirm our theoretical results with experimental ones.

\section{REFERENCES}

[1] P. Cortés, M. P. Kazmierkowski, R. M. Kennel, D. E. Quevedo, and J. Rodriguez, "Predictive Control in Power Electronics and Drives," Industrial Electronics, IEEE Transactions on, vol. 55, no. 12, pp. 43124324, dec 2008.

[2] S. Kouro, P. Cortés, R. Vargas, U. Ammann, and J. Rodriguez, "Model Predictive Control-A Simple and Powerful Method to Control Power Converters," Industrial Electronics, IEEE Transactions on, vol. 56, no. 6 , pp. 1826-1838, jun 2009.

[3] A. Linder, R. Kanchan, R. Kennel, and P. Stolze, "Model-Based Predictive Control of Electrical Drives," Cuvillier Verlag Göttingen, 2010.

[4] G. Goodwin, M. Seron, and J. De Doná, "Constrained control and estimation: an optimisation approach," Springer Verlag, 2005.

[5] H. Khalil, Nonlinear Systems (3rd Edition). Prentice Hall, 2001.

[6] J. Rawlings and D. Mayne, Model Predictive Control: Theory and Design. Nob Hill Publishing, 2009.

[7] T. Geyer, G. Papafotiou, and M. Morari, "Model Predictive Control in Power Electronics: A Hybrid Systems Approach," in 44th IEEE Conference on Decision and Control. IEEE, 2005, pp. 5606-5611.

[8] H. Lin and P. J. Antsaklis, "Stability and Stabilizability of Switched Linear Systems: A Survey of Recent Results," Automatic Control, IEEE Transactions on, vol. 54, no. 2, pp. 308-322, feb 2009.

[9] R. Kennel, A. Linder, and M. Linke, "Generalized predictive control (GPC)-ready for use in drive applications?" in Power Electronics Specialists Conference, 2001. PESC. 2001 IEEE 32nd Annual, 2001, pp. 1839-1844.

[10] D. Mayne, J. Rawlings, and C. Rao, "Constrained model predictive control: Stability and optimality," Automatica, vol. 36, pp. 789-814, 2000.

[11] R. P. Aguilera and D. E. Quevedo, "On the stability of MPC with a Finite Input Alphabet," in 18th World Congress of the International Federation of Automatic Control, Milano, aug 2011. 